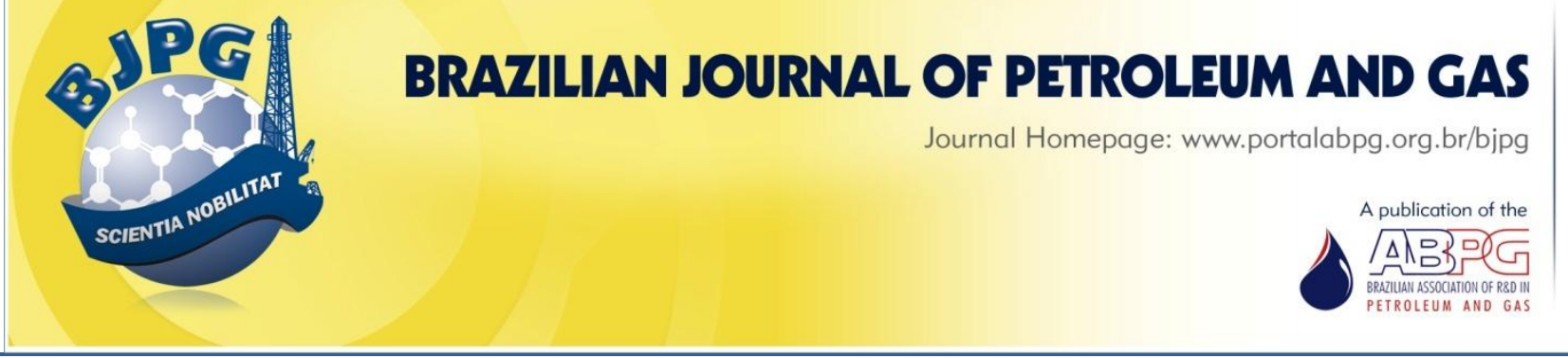

\title{
POLYMER APPARENT VISCOSITY DEPENDENCE ON INACCESSIBLE PORE VOLUME: LABORATORY AND FIELD STUDIES OF ITS INFLUENCE ON ENHANCED OIL RECOVERY
}

\author{
${ }^{\text {a }}$ Ferreira, V. H. S. ${ }^{1} ;{ }^{\text {a }}$ Moreno, R. B. Z. L. \\ ${ }^{a}$ University of Campinas (UNICAMP), School of Mechanical Engineering, Department of Energy, Campinas - SP - Brazil
}

Received: 20.08.2018 / Revised: 14.10.2018 / Accepted: 24.10.2018 / Published on line: 10.01.2019

\begin{abstract}
The successful use of polymer-enhanced oil recovery observed in the last two decades is leading to effective field implementations. One of the reasons for such positive results in polymer flooding is the integration between laboratory-measured properties and reservoir simulation. Recent reports show that inaccessible pore volume (IAPV) plays a significant role in the apparent viscosity of random coil polymers, such as hydrolyzed polyacrylamide (HPAM). This paper assesses both direct and indirect impacts of IAPV on polymer flooding. This investigation relies on laboratory and field-scale simulations of polymer flooding using CMG-STARS. Simulation cases review the effects of IAPV on production indicators in direct and indirect forms. This study compares laboratory-scale simulations with experimental results and uses quality indicators to evaluate the history matching of both approaches. It relies on a modified benchmark field case to study both approaches in comparison to waterflooding and idealized polymer flooding. Results indicate that IAPV has a small direct impact on production curves. However, data show significant indirect implications of the IAPV on recovery. This effect occurs because the apparent viscosity of HPAM has a direct relationship with IAPV. Although the simulation results were consistent with current literature, the results obtained through the experiments indicate that the most realistic simulation case is achieved when considering the impact of IAPV on polymer viscosity.
\end{abstract}

KEYWORDS

polymer flooding; inaccessible pore volume; reservoir simulation; laboratory scale; field scale

\footnotetext{
${ }^{1}$ To whom all correspondence should be addressed.

Address: University of Campinas, Department of Energy, School of Mechanical Engineering, Rua Mendeleyev, 200 - Cidade Universitária, Campinas - SP - Brazil.

ZIP Code: 13083-860 | e-mail: ferreira.vitorh@gmail.com

doi:10.5419/bjpg2018-0019
} 


\section{INTRODUCTION}

Enhanced oil recovery (EOR) by polymer flooding consists of the addition of water-soluble polymers in the injection water. The dissolution of these polymers increases the viscosity of the displacing fluid, improving its areal and vertical sweep efficiencies (Sorbie, 1991). However, polymer flooding is subject to various complex phenomena, such as non-Newtonian viscosity, inaccessible pore volume (IAPV), and retention. Literature reports several successful polymer flooding field implementations in the last decades (Delamaide, 2014; Standnes \& Skjevrak, 2014). One of the reasons for this success is the integration between laboratory-measured properties and reservoir simulations. Reservoir simulators are computational tools largely used in the petroleum industry for history matching and behavior forecasting (Aziz \& Settari, 1979). Reservoir simulators are very useful for risk assessment, production strategy definition, and decision-making. Typically, the input data in a reservoir simulation are a combination of field and laboratory data. Due to the complex nature of EOR processes, the operator must be careful on the correct representation of the phenomena observed in the laboratory for the simulation to be accurate.

The IAPV is a phenomenon characteristic of the polymer when flowing through a porous medium. Dawson and Lantz (1972) reported that this phenomenon results of polymer molecules transport through a smaller pore volume when compared with smaller molecules. Due to this smaller accessible pore volume, the polymer molecules are transported faster through the porous medium than smaller molecules. Dominguez and Willhite (1977) defined that the IAPV is a consequence of the size of the polymer molecules being too big to fit some pores, making some pores inaccessible to polymer molecules. In contrast, Sorbie (1990) explained that this phenomenon is due to a thin layer of polymer-free fluid near the pore surface, which is named the depleted layer. Frequently, reports consider the IAPV as having a small impact on the flow of polymers in a porous medium, especially in twophase flow (Ferreira \& Moreno, 2017a; Lamas et al., 2016). However, recent studies point to a correlation between IAPV and the apparent viscosity of polymer solutions flooding through a porous medium (Seright et al., 2011; Stavland et al., 2010). The injected fluid viscosity is a key parameter on any EOR process, especially on a mobility control one, such as polymer flooding.

The objective of this paper is to quantify the direct and indirect impacts of IAPV in the oil production indicators through reservoir simulations. The direct impact is due to the accelerated transport of polymer through the porous medium, and the indirect one is due to the effect of IAPV on the viscosity of the injected fluid. This study is done both in laboratory and field scale simulations.

\section{MATERIALS AND METHODS}

This work consists of two parts: laboratory (L) and field (F) scale simulations of oil recovery aiming to study polymer flooding. For the field scale, a comparative study analyzed waterflooding, idealized polymer, and different polymer modeling regarding IAPV and in-situ viscosity. Conversely, for the laboratory scale, a real experiment was used to compare different polymer models. Therefore, waterflooding (WF) and idealized polymer flooding (IPF) were only studied in the field scale. We considered three cases to model polymer observing IAPV and in-situ viscosity. Case 1 was a polymer with no IAPV (IAPV=0). Case 2 considered an IAPV of $10 \%$, but with no IAPV effect on the viscosity. Case 3 considered an IAPV of $10 \%$ and its correspondent effect on viscosity. Table 1 summarizes the cases studied in this paper.

The polymer referenced to in the study was a synthetic terpolymer composed of polyacrylamide, acrylic acid, and ATBS (Flopaam $5115 \mathrm{SH}$ ) with a molecular weight between 14 and 16 million Daltons, and $15 \%$ hydrolysis degree (Ferreira \& Moreno, 2017b; Silveira et al., 2018).

In Table 1, the bulk is the polymer viscosity observed in a rheometer while the in-situ one is the viscosity the polymer experiences as it flows through a porous medium. In this case, a Haake Mars III rheometer was used to measure the bulk viscosity $\left(\eta_{\text {bulk }}\right)$ and the results were reported previously by Silveira et al. (2016). The study used Eq. 1 (a model developed by Stavland et al., 2010), considering $10 \%$ IAPV, to obtain the in-situ viscosity $\left(\eta_{\text {is }}\right)$. 
Table 1. Summary of the differences between the input data for all the cases.

\begin{tabular}{ccccc}
\hline \hline Case & Scale & Flooding & IAPV & Viscosity \\
\hline \hline L1 & Laboratory & Polymer & 0 & Bulk \\
L2 & Laboratory & Polymer & $10 \%$ & Bulk \\
L3 & Laboratory & Polymer & $10 \%$ & In-situ \\
FWF & Field & Water & 0 & Newtonian \\
FIPF & Field & Idealized Polymer & 0 & Newtonian \\
FPF1 & Field & Polymer & 0 & Bulk \\
FPF2 & Field & Polymer & $10 \%$ & Bulk \\
FPF3 & Field & Polymer & $10 \%$ & In-situ \\
\hline \hline
\end{tabular}

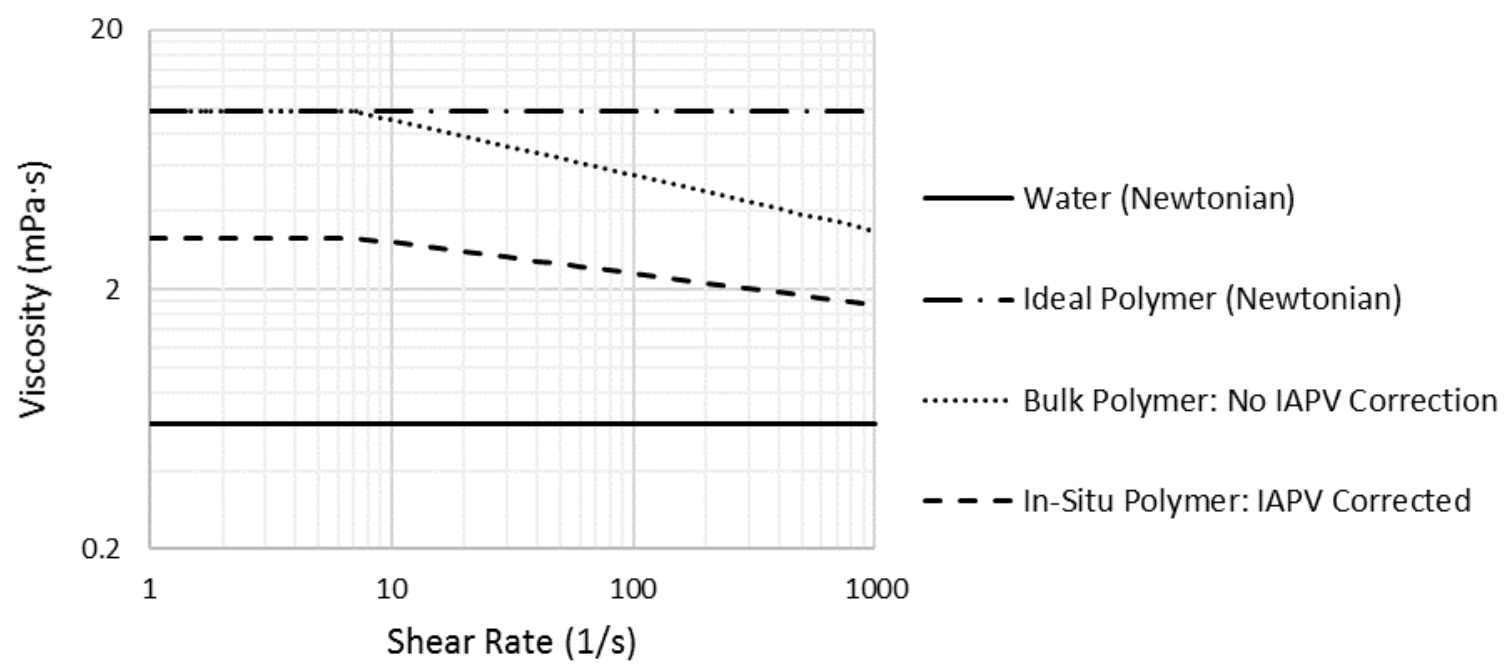

Figure 1. Viscosity versus shear rate for the cases studied (bulk viscosity based on Silveira et al. (2016); in-situ viscosity based on Ferreira \& Moreno (2017b)).

$\eta_{i s}=\frac{\eta_{b u l k} \cdot(1-I A P V)^{-2}}{1+\left[(1-I A P V)^{-2}-1\right] \cdot \sqrt{\eta_{b u l k} / \mu_{w}}}$

Where $\mu_{\mathrm{w}}$ is the water viscosity.

Ferreira and Moreno (2017b) verified that the model presented by Eq. 1 matched the in-situ viscosity of this specific HPAM-ATBS. Figure 1 shows bulk and in-situ polymer viscosities, as well as the water viscosity and ideal polymer viscosity.

One can see in Figure 1 that, even for the small $10 \%$ of IAPV, a significant difference between bulk and in-situ viscosities can occur. For low shear rates, the viscosity reduction is as high as $67 \%$.

The oil-water relative permeability curves used for all cases are depicted in Figure 2.

The unsteady state method, known as JBN, was

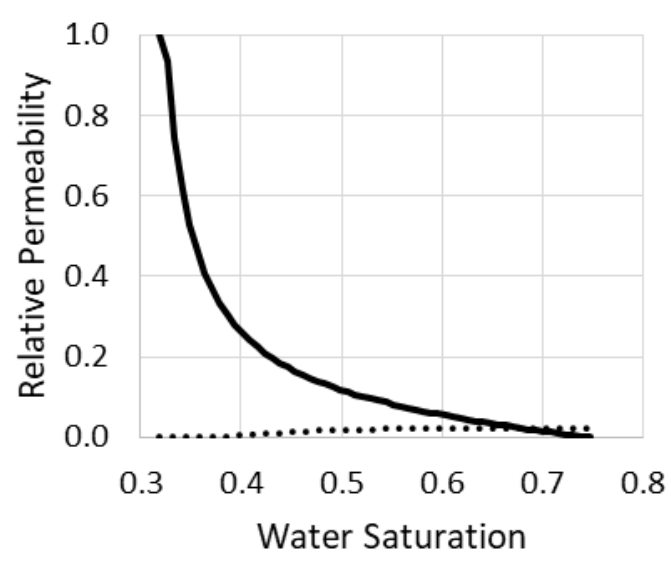

Oil Phase $\quad \cdots . .$. Water Phase

Figure 2. Oil-Water relative permeability curves (based on Silveira (2017)). 


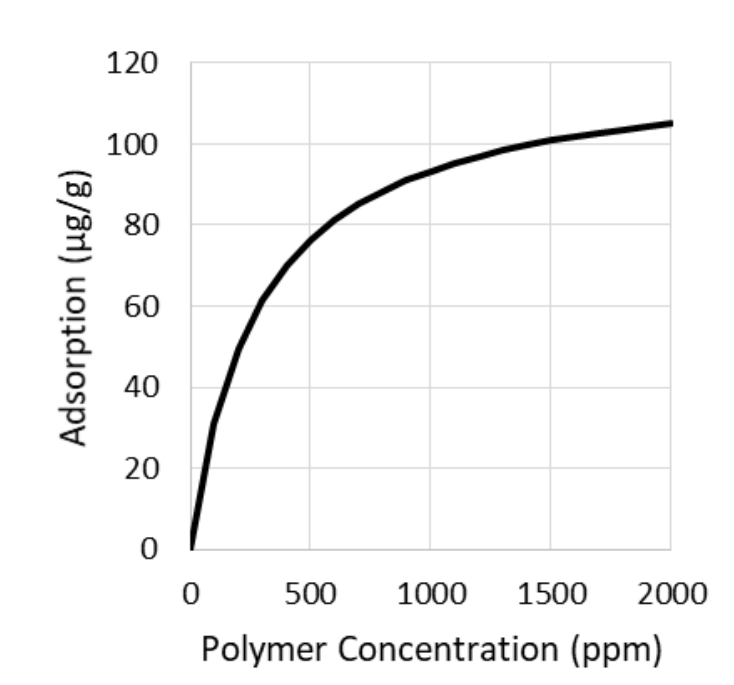

Figure 3. Polymer adsorption curve (based on Ferreira \& Moreno (2017b)).

used to measure the relative permeabilities experimentally (Johnson et al., 1959). The terminal points for residual oil saturation and irreducible water saturation correspond to $25.2 \%$ and $31.9 \%$, respectively.

The idealized polymer consisted of viscosified water with no adsorption, no inaccessible pore volume, unitary residual resistance factor, and Newtonian viscosity. For the other polymer cases (field and laboratory), the residual resistance factor was 1.04 (measured in the laboratory by Silveira et al., 2018). Figure 1 presents the viscosity, and Figure 3 shows the adsorption.

All polymer flooding simulations used the injected polymer concentration of 2000 ppm, based on the work of Silveira et al. (2018). This work considered no polymer degradation to simplify the model. This decision took into account that the degradation should not have any effect in the laboratory-scale simulations. It also considered the availability of a previous work assessing the impact of polymer degradation in field-scale simulations (Lamas et al., 2016). The methodology of this work represents a part of the workflow proposed by Ferreira and Moreno (2018). It highlights the integration among single-phase and two-phase core flooding laboratory experiments and simulation models for core scale and full field scale representations. This work focuses on the core and field scale simulations, therefore the laboratory and reservoir data were taken from the literature. Figure 4 presents the workflow followed to develop this work.

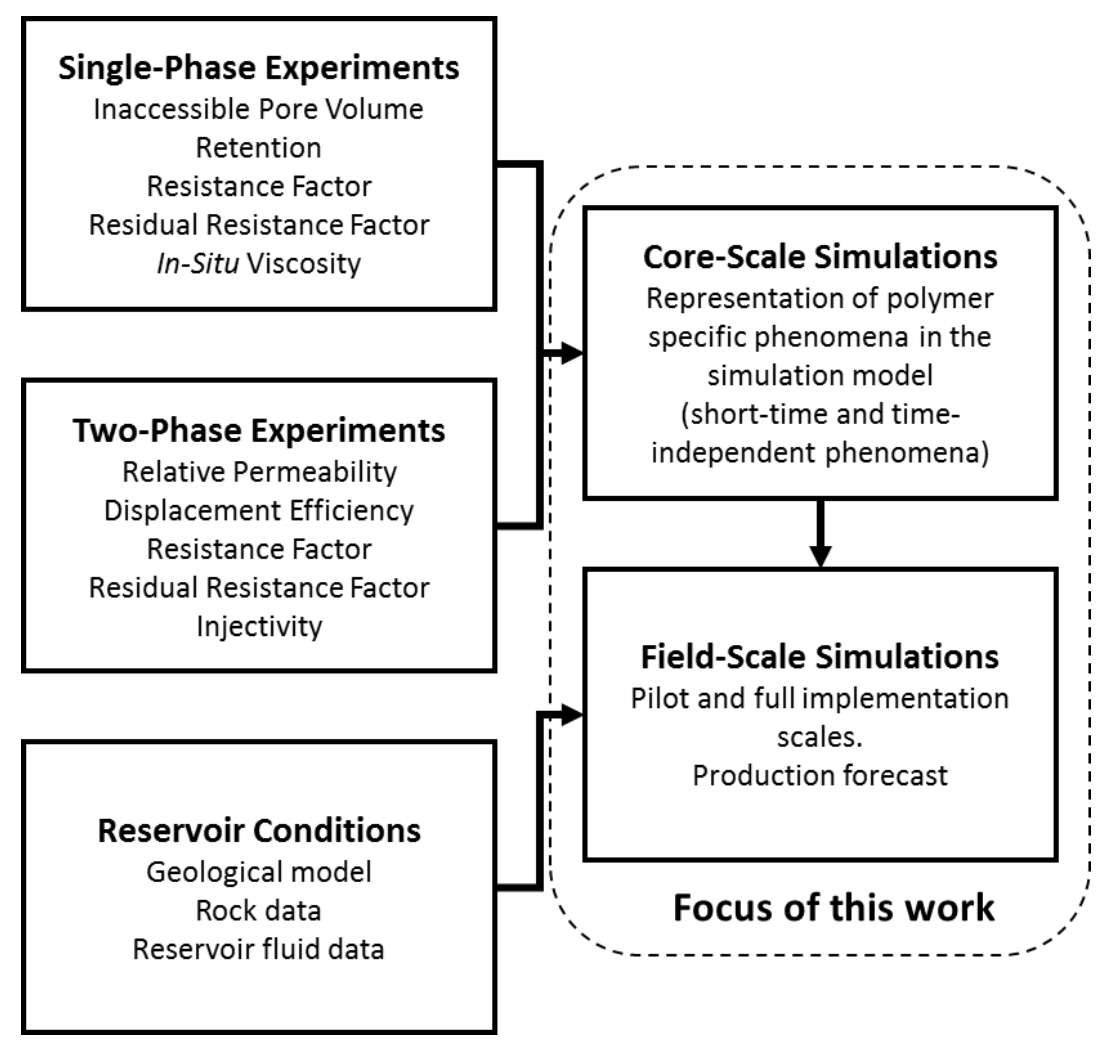

Figure 4. Workflow of this work. 
Table 2. Summary of the core properties, and initial and boundary conditions for laboratory scale simulation cases (L1, L2, and L3).

\begin{tabular}{|c|c|c|c|}
\hline Core Length & $6.1 \mathrm{~cm}$ & Initial Oil Saturation & $68.1 \%$ \\
\hline Area Open to Flow & $11.15 \mathrm{~cm}^{2}$ & Initial Water Saturation & $31.9 \%$ \\
\hline Porosity & $31.3 \%$ & Initial Reservoir Pressure & $101 \mathrm{kPa}$ \\
\hline Absolute Permeability & $3.877 \mu \mathrm{m}^{2}$ & Injection Flow Rate & $12.2 \mathrm{~mm}^{3} / \mathrm{s}$ \\
\hline Temperature & 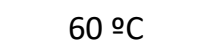 & Production Pressure & $101 \mathrm{kPa}$ \\
\hline Oil Viscosity & $180.39 \mathrm{mPa} \cdot \mathrm{s}$ & & \\
\hline
\end{tabular}

The methodology used in this work to build the simulation model for polymer core flooding representation is based on Zampieri (2017). This study used the CMG-STARS reservoir simulator. Following, the models of laboratory and field cases are available in each respective section.

\subsection{Laboratory scale model description}

The laboratory scale model represented a twophase core flooding experiment of secondary oil recovery by polymer flooding. Silveira et al. (2018) reported the experimental results. Therefore, the combination of experimental and simulation results led to the evaluation of the quality of the match in the three laboratory scale simulation cases.

Table 2 summarizes the simulation input data of the laboratory scale reservoir, as well as the initial and boundary conditions. These data were used in conjunction with those presented in Table 1 and Table 2 to build the laboratory model.

The simulation grid was one-dimensional with a square cross-sectional area (Figure 5). The core used in the experiment was a cylinder, and the simulation model used the same area open to flow. The simulation used the constant porosity and permeability determined by Silveira et al. (2018). The injector well was constrained to constant injection flow rate and the producer well produced under constant pressure. The simulation was isothermal.

\subsection{Field scale model description}

The field scale model was based on the benchmark case UNISIM-I-M (Avansi \& Schiozer, 2015; Gaspar et al., 2016). The UNISIM-I-M is a field model based on the Namorado Field, Campos
Basin, in Brazil. This model was created to serve as a benchmark case for reservoir management and decision making for the period after the initial strategy, and almost all the infrastructure had been defined. However, this work uses a modified model to represent the field case being analyzed.

UNISIM-I-M has a waterflooding strategy to recover light oil ( $1.11 \mathrm{mPa} \cdot \mathrm{s}$ at the reservoir conditions) in a reservoir with an average horizontal permeability of $283 \mu \mathrm{m}^{2}$. Aiming to bring UNISIM-I-M reservoir closer to a polymer-flooding candidate, some modifications to the reservoir properties took place. Such modifications are listed in Table 3.

The modified model was ported to the CMGSTARS, since the UNISIM-I-M was implemented in CMG-IMEX. Reservoir temperature, oil viscosity, relative permeabilities, residual oil saturation, connate water saturation, and average absolute horizontal permeability were all modified to match

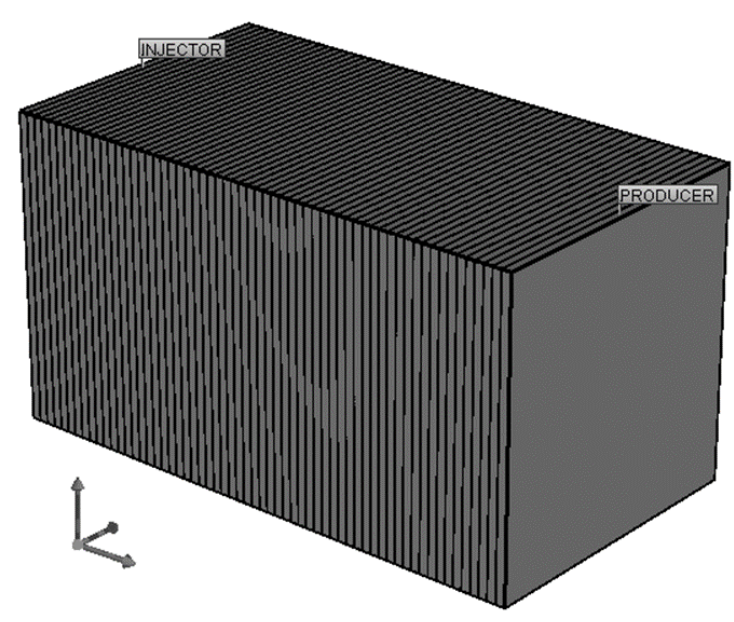

Figure 5. Laboratory reservoir model. 
Table 3. Summary of modifications between the original UNISIM-I-M model and the one used in this study.

\begin{tabular}{lccc}
\hline \hline \multicolumn{1}{c}{ Property } & Unit & Original & Modified \\
\hline \hline Simulator & - & IMEX & STARS \\
Reservoir Temperature & ${ }^{\circ} \mathrm{C}$ & 80 & 60 \\
Live Oil Viscosity & $\mathrm{mPa} \cdot \mathrm{s}$ & $\sim 1.11$ & 180.39 \\
Relative Permeabilities & - & & Figure 6 \\
Residual Oil Saturation & - & $18 \%$ & $25.2 \%$ \\
Irreducible Water Saturation & - & $17 \%$ & $31.9 \%$ \\
Average Absolute Horizontal Permeability & $\mu \mathrm{m}^{2}$ & 0.283 & 3.877 \\
Average Absolute Vertical Permeability & $\mathrm{\mu m}^{2}$ & 0.119 & 0.775 \\
\hline \hline
\end{tabular}

values measured in the experiment. The average absolute vertical permeability was changed to correspond to $20 \%$ of the horizontal one. It is worth mentioning that the oil viscosity was modified to match the experimental value in the correspondent reservoir conditions. The PVT data for the field oil was based on the work of de Ghetto et al. (1995), and no gas was formed in the reservoir during the entire simulation. The initial oil and water saturations were adjusted to correspond to laboratory-measured conditions (i.e., terminal points presented in the modified case of Figure 6. All the polymer properties were modeled as in the laboratory scale model.

It is important to mention that this paper is not a production optimization study, nor a risk assessment analysis. The production strategy and restrictions defined in the original UNISIM-I-M were unchanged from the benchmark model
(IMEX) to our modified model (STARS). Also, the oil recovery performance was performed to analyze produced oil and injected/produced water (i.e., no net present value analysis).

A waterflooding case (FWF) and an ideal polymer case (FIPF) were simulated to serve as comparative models to the different modeling approaches of the polymer flooding considering IAPV and in-situ viscosity (FPF1, FPF2, FPF3).

Table 4 summarizes other relevant properties for the field model and Figure 7 presents a top view of the field with the overlapping well position.

\subsection{History matching quality indicators}

The analysis of the quality of the history matching between simulation and observed laboratory data used three different quality indicators: normalized absolute linear distance

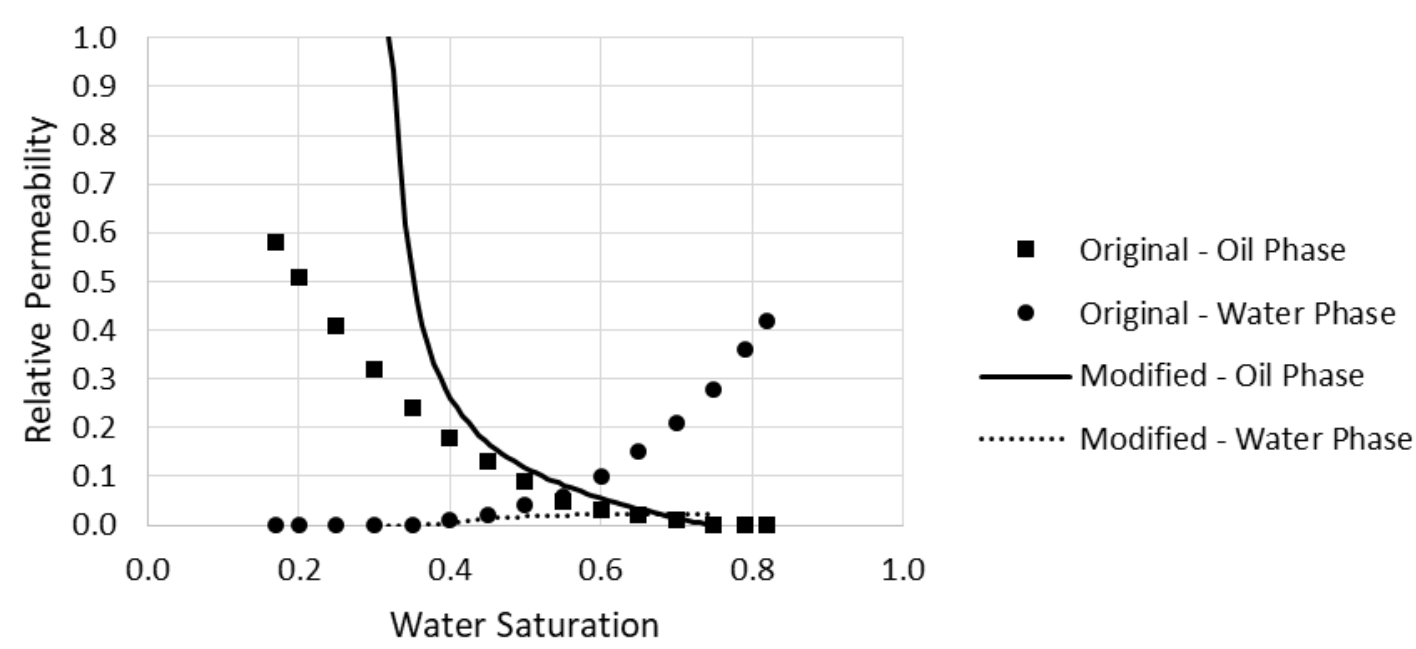

Figure 6. Relative permeabilities of the oil and water phases for the original UNISIM-I-M model and the modified model. 
Table 4. Summary of other relevant simulation model properties.

\begin{tabular}{ccc}
\hline \hline \multicolumn{2}{c}{ Grid Blocks (i, j, k) } & \\
Active Blocks & & $31,58,20$ \\
Average Effective Porosity & & 36380 \\
& Time & $29.4 \%$ \\
\hline \multirow{2}{*}{ Primary Oil Production } & Producers & 3 Years and 2 Months \\
& Injectors & Nortical \\
\hline \multirow{2}{*}{ Secondary Oil Recovery ${ }^{1}$} & Time & 32 years and 9 months ${ }^{2}$ \\
& Producers & 4 Vertical + 10 Horizontal \\
& Injectors & 11 Horizontal \\
\hline \hline
\end{tabular}

1- Waterflooding for the FWF case and polymer flooding for cases FIPF, FPF1, FPF2, and FPF3;

2 - Started after the primary production period.

(NALD), Eq. 2, normalized quadratic distance (NQD), Eq. 3, and normalized quadratic distance including signal (NQDS), Eq. 4.

$$
\begin{aligned}
& N A L D=\left|\operatorname{Sim}_{i}-O b s_{i}\right| / O b s_{i} \\
& N Q D=Q D / A Q D \\
& N Q D S=N Q D \cdot L D /|L D|
\end{aligned}
$$

Where $\operatorname{Sim}$ is the simulated data, Obs is the observed laboratory data, $N$ is the number of observed data, $Q D$ is the quadratic distance, $A Q D$ is the acceptable quadratic distance, and $L D$ is the linear distance. Equations 5, 6, and 7 define the $Q D, A Q D$, and $L D$, respectively.

$Q D=\sum_{i=1}^{N}\left(\operatorname{Sim}_{i}-O b s_{i}\right)^{2}$

$$
A Q D=\sum_{i=1}^{N}\left(T o l \cdot O b s_{i}+C_{p}\right)^{2}
$$

$$
L D=\sum_{i=1}^{N}\left(\operatorname{Sim}_{i}-O b s_{i}\right)
$$

Where $T o l$ is a tolerance related to the observed data, and $C_{p}$ is a constant to prevent error when the observed data is close to zero. Maschio and Schiozer (2016) stated that there are no specific rules to determine $T o l$ and $C_{p}$, so these parameters should be chosen on a case-by-case basis, depending on the uncertainty associated with the observed data.

According to Maschio and Schiozer (2016), NQD is a good quality indicator because: 1 . it is dimensionless and considers the reliability of the

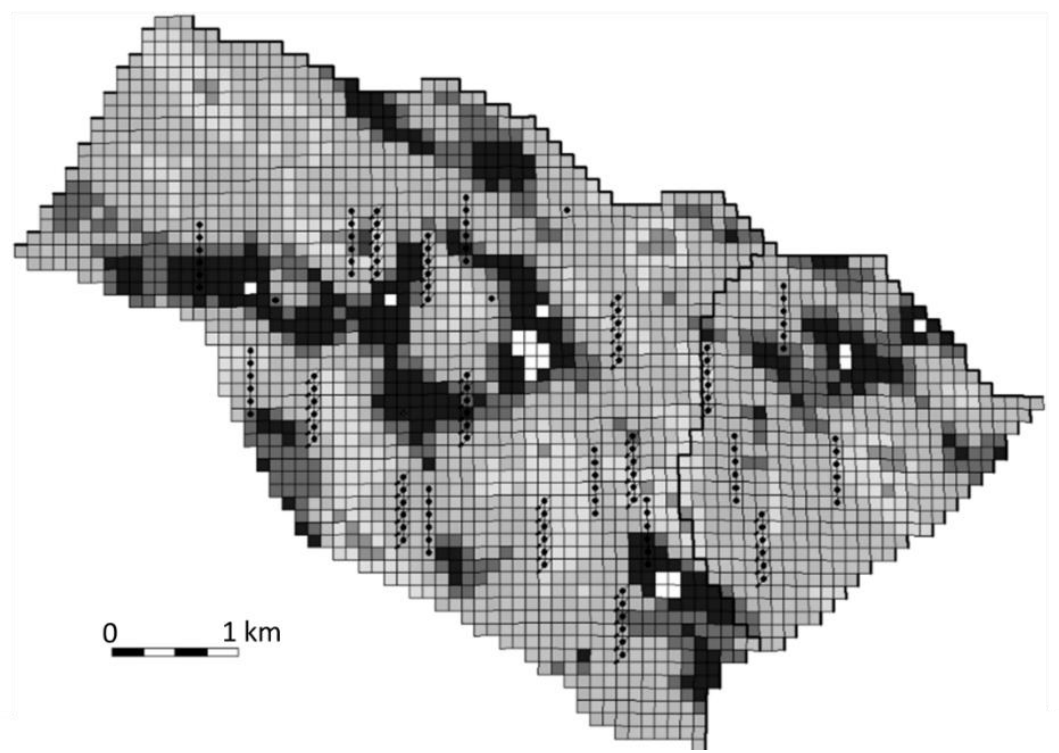

Figure 7. Overlapped disposition of the wells in the field case study (adapted from Gaspar et al. (2016)) 
Table 5. History matching quality indicators for the laboratory scale simulations.

\begin{tabular}{ccccc}
\hline \multirow{2}{*}{ Production Indicator } & & Case \\
& & L1 & L2 & L3 \\
\hline \multirow{2}{*}{ Differential Pressure } & NQD & 63.15 & 64.07 & 0.83 \\
$(\Delta \mathrm{p})$ & NQDS & 63.15 & 64.07 & 0.83 \\
& Average NALD & $12.1 \%$ & $12.3 \%$ & $1.0 \%$ \\
\hline \multirow{2}{*}{ Oil Recovery Factor } & NQD & 1.59 & 1.61 & 0.26 \\
$($ FR) & NQDS & 1.59 & 1.61 & -0.26 \\
& Average NALD & $13.6 \%$ & $13.7 \%$ & $7.4 \%$ \\
\hline \multirow{2}{*}{ Cumulative Oil Production } & NQD & 3.09 & 3.11 & 0.50 \\
$($ Np) & NQDS & 3.09 & 3.11 & -0.50 \\
& Average NALD & $13.5 \%$ & $13.6 \%$ & $7.4 \%$ \\
\hline \multirow{2}{*}{ Cumulative Water Production } & NQD & 0.97 & 0.97 & 0.79 \\
$($ Wp) & NQDS & 0.97 & 0.97 & 0.79 \\
& Average NALD & $2.9 \%$ & $3.0 \%$ & $1.0 \%$ \\
\hline \multirow{2}{*}{ Cumulative Water-Oil Ratio } & NQD & 3.19 & 3.20 & 0.65 \\
$($ WOR) & NQDS & -3.19 & -3.20 & -0.65 \\
& Average NALD & $12.5 \%$ & $12.9 \%$ & $4.5 \%$ \\
\hline \multirow{2}{*}{ Cumulative Water Cut } & NQD & 0.06 & 0.07 & 0.01 \\
(Wcut) & NQDS & 0.06 & -0.07 & 0.01 \\
& Average NALD & $2.4 \%$ & $2.5 \%$ & $0.7 \%$ \\
\hline \hline
\end{tabular}

observed data in the calculation; 2 . it allows the combination of different kinds and magnitudes of data; 3 . it makes the analysis of large quantities of data assessable. Maschio and Schiozer (2016) also explained that NQDS is used to assess if the simulated data overestimates or underestimates the observed data.

The use of NALD also provides a dimensionless quality of match indicator with the advantage of using the raw values of simulation and observed data. This indicator provides a clean result, as it is not biased by $T o l$ and $C_{p}$, and can be expressed as a percentage of difference relative to the observed data. Nevertheless, NALD is not very suited for data that are very close to zero and can lead to an undefined value when the observed data is equal to zero (i.e., division by zero).

\section{RESULTS AND DISCUSSIONS}

The results are divided into the history matching analysis of the laboratory experiment and the study of the effects of the different polymer modeling in a field model.

\subsection{Laboratory scale}

Figure 8 and Figure 9 present the results of the core flooding experiment (adapted from Silveira et al., 2018) and the simulation models (L1, L2, and L3) along with the point-to-point NALD. Table 5 summarizes the history matching quality indicators.

The direct impact of the IAPV, i.e., lower effective porosity, is visible when comparing cases $\mathrm{L} 1$ and $\mathrm{L} 2$. Analysis of the quality indicators reveals that this direct impact on the production parameters (FR, Np, Wp, WOR, and Wcut) is small, corroborating with current literature (Ferreira \& Moreno, 2017a; Lamas et al., 2016). The average NALD difference between L1 and L2 was lower than $0.5 \%$ for all the parameters analyzed. Similar results were observed for the NQD and NQDS. Careful examination of the quality indicators reveals that the consideration of the IAPV alone led to worse history matches. Nevertheless, in light of the small difference in results, one may consider it to be circumstantial.

Comparison between cases $L 2$ and $L 3$ reveal the indirect impact of the IAPV, i.e., the reduction of apparent viscosity. The history matching quality improved significantly for all the parameters analyzed when considering the IAPV effect on viscosity, most notably for the differential pressure $(\Delta p)$. Average NALD reductions as high as $11 \%$ were observed from case L2 to case L3.

An important aspect of the improvement of the history matching arises when analyzing the pointto-point NALD presented in Figure 8 and Figure 9. 

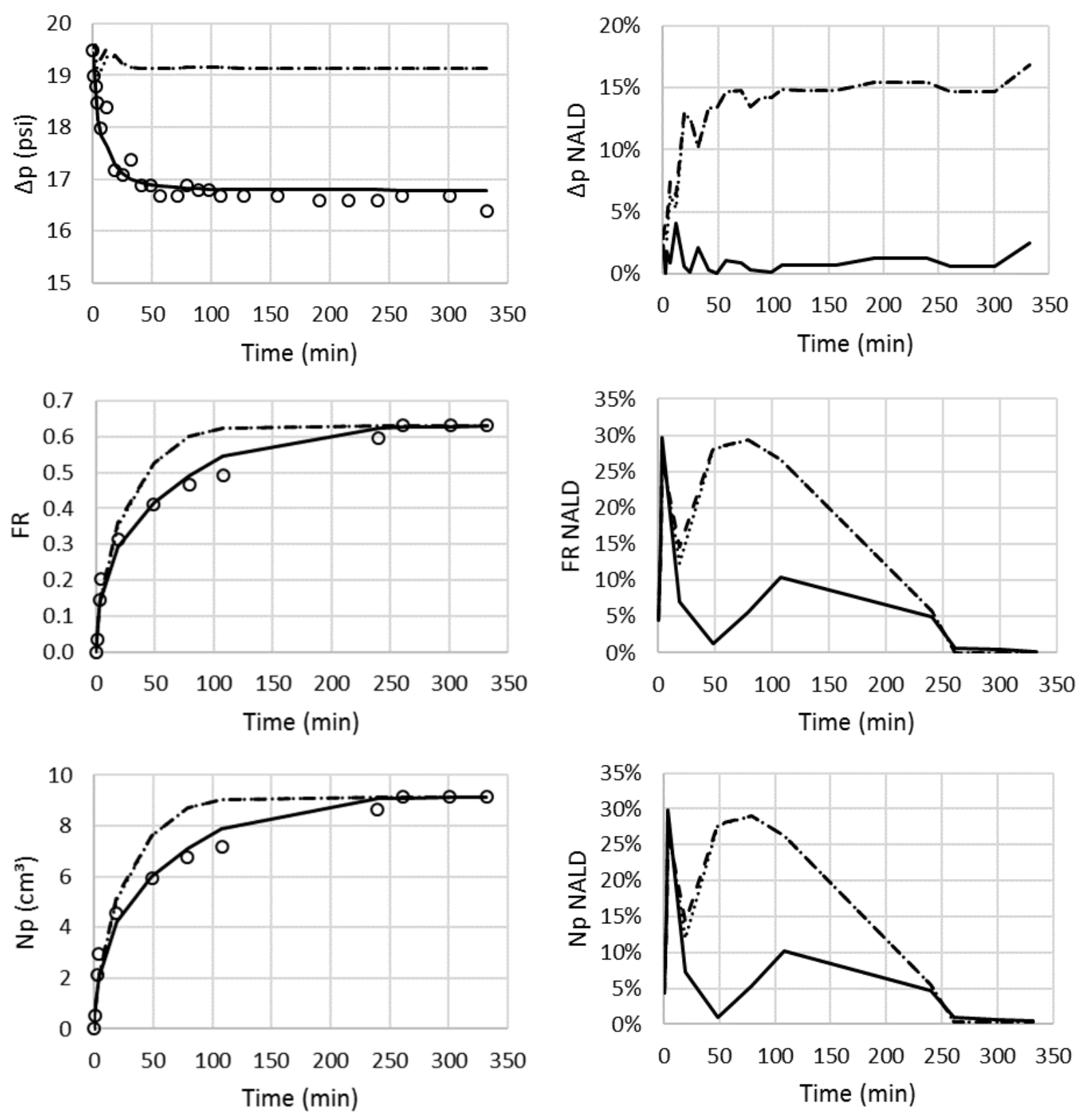

○ Experimental

$\mathrm{L} 1 \quad--\mathrm{L} 2$ L3

Figure 8. Experimental and simulated results (left) and NALD (right) for the differential pressure across the core $(\Delta p)$, oil recovery factor (FR), and cumulative oil production ( $\mathrm{Np}$ ). Table 1 summarizes the simulation models.

In those figures, one can see that the consideration of IAPV effect on viscosity leads to an improved history matching in the early simulation times (i.e., transient regimes). When steady state is reached (i.e., late simulation times), the results from L1, L2, and $\mathrm{L} 3$ tend to stabilize at the same value, with exception to differential pressure.

The cases that consider bulk viscosity (L1 and L2) tend to overestimate the polymer performance, that is, L1 and L2 overestimate the FR and underestimates WOR. This unrealistic improvement in performance is a direct result of the higher viscosity value taken into account for the aqueous phase, as presented in Figure 10.

The difference in steady-state differential pressure, observed in Figure 8 , is also a direct result of the viscosity of the water phase.

The indicators presented in Table 5 reveal that the case $L 3$ caused all the quality indicators to approach zero, in which zero indicates a perfect match between experiment and simulation. The 

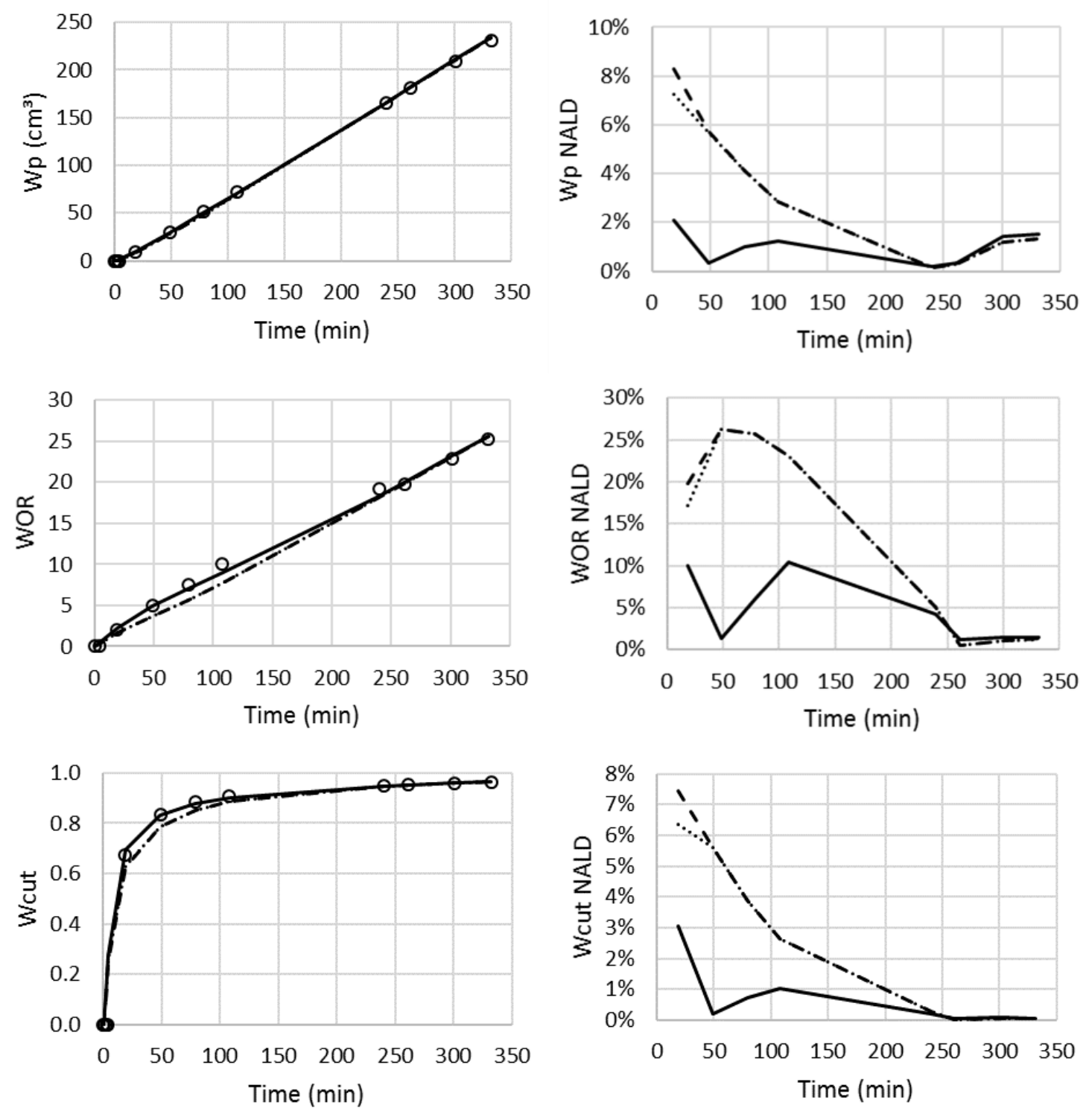

○ Experimental

$--\mathrm{L} 2-\mathrm{L3}$

Figure 9. Experimental and simulated results (left) and NALD (right) for the cumulative water production (Wp), cumulative water-oil ratio (WOR), and cumulative water cut (Wcut). Table 1 summarizes the simulation models.

cases L1 and L2 overestimated all the production indicators, and the case L3 resulted in significant reductions in NQD, NQDS, and average NALD. The NQD and NQDS reductions were up of 63.32, while the highest average NALD reduction was of $11.3 \%$. Therefore, one can conclude that consideration of IAPV combined with its effect in on in-situ viscosity, as evaluated in the case $\mathrm{L} 3$, is the most realistic approach to polymer flooding simulation.

\subsection{Field scale}

For the field-scale study, the waterflooding (FWF) and the ideal polymer flooding (FIPF) represent the performance limits to the cases FPF1,

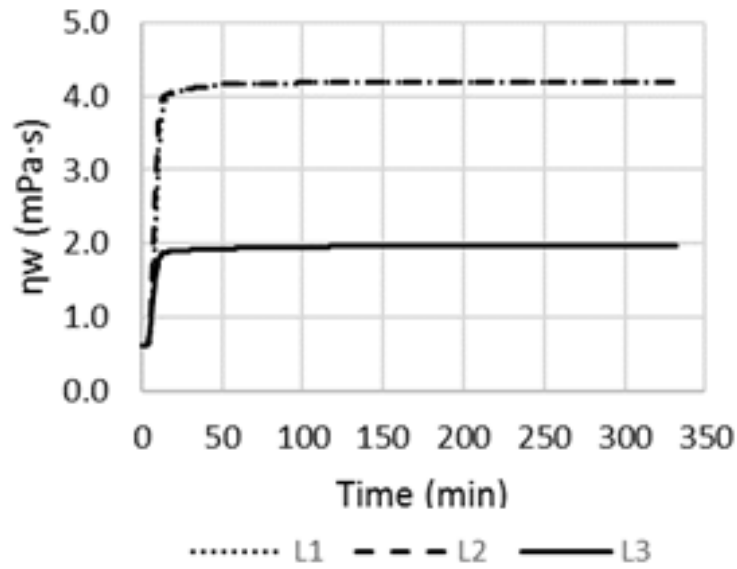

Figure 10. Aqueous phase apparent viscosity in block 31 (middle of the core) for the laboratory scale study. Table 1 summarizes the simulation models. 

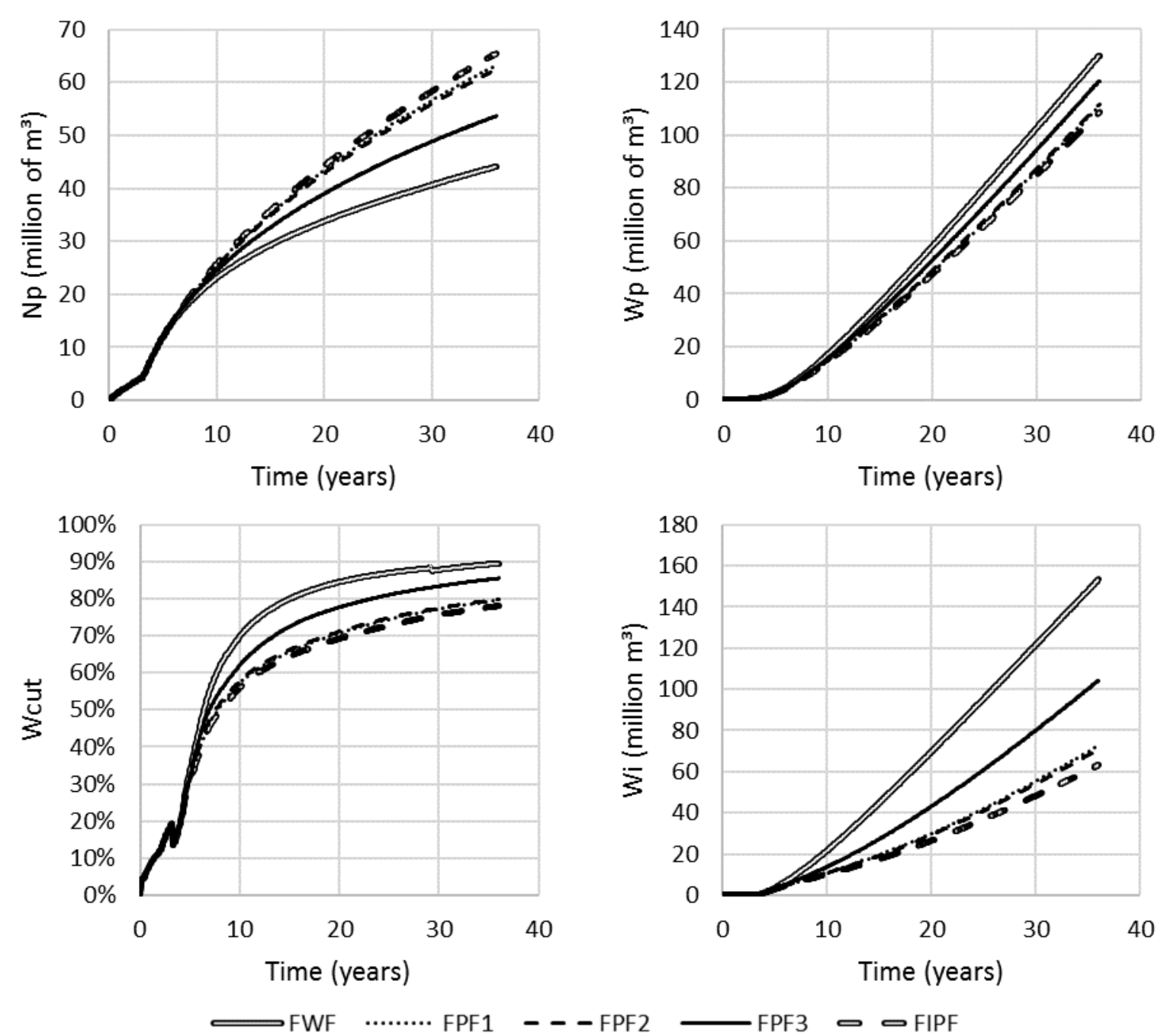

Figure 11. Field simulation results of cumulative oil production ( $\mathrm{Np})$, cumulative water production (Wp), cumulative water cut (Wcut), and cumulative water injection (Wi). Table 1 summarizes the simulation models.

FPF2, and FPF3. In Figure 11, one can see that the injection and production performance of FPF1, FPF2, and FPF3 models stand between FWF and FIPF.

The ideal polymer is the most optimistic case, i.e., the one with the highest produced oil volume, and lowest produced and injected water volumes. The waterflooding, on the other hand, is the least optimistic one. The cases considering bulk viscosity (FPF1 and FPF2) had a performance close to the ideal polymer. The polymer flooding case considering in-situ viscosity (FPF3) stands close to the halfway mark between the ideal polymer and waterflooding performances.

It is worth noting that a real field analysis regarding selection and/or risk assessment of a production strategy would have to take into account the net present value (NPV) associated with each method, but this falls outside the scope of this paper.

The differences aforementioned are also present in the analysis of the injection and production parameters at the end of the simulation time, as presented in Table 6.

The inclusion of the shear-thinning bulk viscosity model (FPF1 and FPF2) resulted in worse performance than the idealized Newtonian viscosity polymer (FIPF). The polymer solution flows most of the time in regions far from injection and production wells, i.e., deep within the reservoir. The maximum value for the water phase shear rate far away from wells is around $10 \mathrm{~s}^{-1}$, which results in apparent viscosities above $9 \mathrm{mPa} \cdot \mathrm{s}$ for FPF1 and FPF2. These apparent viscosities are only $7 \%$ lower than the idealized polymer viscosity. However, the inclusion of the shear thinning bulk 
Table 6. Differences among all the cases relative to the idealized polymer flooding field case (FIPF). Injection and production simulation results for the end of simulation time.

\begin{tabular}{ccccc}
\hline \hline & FWF & FPF1 & FPF2 & FPF3 \\
FR & $-32.6 \%$ & $-3.4 \%$ & $-4.5 \%$ & $-18.1 \%$ \\
Np & $-32.6 \%$ & $-3.4 \%$ & $-4.5 \%$ & $-18.1 \%$ \\
Wp & $19.8 \%$ & $2.1 \%$ & $2.7 \%$ & $10.9 \%$ \\
WOR & $77.7 \%$ & $5.6 \%$ & $7.6 \%$ & $35.4 \%$ \\
Wcut & $14.7 \%$ & $2.0 \%$ & $2.3 \%$ & $9.5 \%$ \\
Wi & $142.3 \%$ & $14.9 \%$ & $12.1 \%$ & $64.9 \%$ \\
\hline \hline
\end{tabular}

viscosity resulted in relative differences up to $15 \%$ in the injection/production performance. This difference is due to the polymer behavior near the wells. Even though the polymer solution stays a relatively short time in the high shear rate regions near the wells, the viscosity reduction of FPF1 and FPF2 relative to FIPF are as high as $70 \%$ in these regions. Therefore, based on the observable viscosity behavior, one can say that the idealized Newtonian polymer results in an unrealistic optimistic production performance due to the near-well behavior of the shear-thinning polymer.

The direct impact of the IAPV can be analyzed by the differences in the production performance between FPF1 and FPF2 presented in Figure 11 and Table 6. Considering that the differences are small (within 3\%), the direct impact of the IAPV was as small for the field study, as it was for the laboratory study. The laboratory simulation considering only the direct impact of the IAPV (L2) led to a worse history matching when compared to the case without IAPV consideration (L1). However, the results of the field simulation considering only the direct impact of the IAPV (FPF2) were closer to the case including the in-situ viscosity (FPF3) than to the case without IAPV consideration (FPF1). Even though the result of FPF2 tends to go in the direction of the FPF3, the results are still very far apart from each other. This way, one can consider that the isolate inclusion of IAPV (i.e., without the in-situ viscosity influence) is unrealistic, as it was in the laboratory scale studies.

The indirect impact of the IAPV can be analyzed by comparing cases FPF2 and FPF3. The relative differences between FPF2 and FPF3 are as high as $47 \%$. Therefore, one can see that the IAPV effect on the in-situ viscosity has a much more meaningful impact on the injection/production performance than the direct IAPV effect. Based on our laboratory scale simulation study and current literature on experimental polymer behavior in porous media (Ferreira \& Moreno, 2017b; Stavland et al., 2010), one can say that FPF3 is the most realistic case studied in this paper and, therefore, it should be used in any polymer flooding modeling or simulation.

The cases that did not consider the IAPV effect on the in-situ viscosity of the polymer (FPF1 and FPF2) had results close to the ideal polymer (FIPF) and far from the more realistic case (FPF3). Therefore, one can conclude that simulation models not considering the in-situ viscosity of the polymer may result in over-optimistic and unrealistic forecasts.

The injection wells were limited by the bottomhole pressure constraint rather than the flow rate one for all flooding cases studied in the field scale. This restriction allowed the case considering the lower polymer viscosity (FPF3) to have better injectivity than FPF1 and FPF2. However, in the cases tested, the injectivity was not a significant problem due to the high permeability of the reservoir. Therefore, better sweep efficiencies of cases FPF1 and FPF2 compensated the loss of injectivity and resulted in a more optimistic production than case FPF3. It is important to highlight, however, that injectivity is a major concern during most polymer flooding evaluations (Delamaide, 2014). So, the consideration of bulk polymer viscosity in field simulations could lead to imprecise injectivity analysis and adverse forecasts in cases of low permeability reservoirs.

\section{CONCLUSIONS}

This paper analyzed the direct and indirect impacts of polymer inaccessible pore volume (IAPV) in reservoir simulation of a polymer flooding. The study was performed through 
simulations of core flooding and modified benchmark field models. The main conclusions are:

- The direct effect of polymer IAPV (i.e., lower effective porosity) was of minor impact for both laboratory and field studies. For the laboratoryscale, the direct effect of IAPV led to average NALD differences lower than $0.5 \%$. For the fieldscale, the differences in the results related to the direct impact of IAPV were within 3\%;

- The indirect effect of polymer IAPV (i.e., reduction of apparent viscosity) was of significant impact for both laboratory and field studies. The average NALD differences when considering the indirect effect of IAPV were as high as $11 \%$ for the laboratory scale. The relative differences in the field scale results were as high as $47 \%$ when considering the indirect impact of IAPV;

- The model considering the IAPV effect on the insitu viscosity (L3) resulted in the best history matching for the laboratory experiment. Notably, the average NALD for the core differential pressure was reduced from $12.3 \%$ to $1 \%$ when considering the IAPV effect on viscosity;

- Consideration of Newtonian polymer behavior in our field scale simulations resulted in an unrealistic optimistic production performance due to the near-well behavior of the shearthinning polymer;

- Consideration of bulk-polymer viscosity in polymer flooding simulations may lead to a poor analysis of the injectivity, and adverse forecasts in low permeability reservoirs.

\section{ACKNOWLEDGMENTS}

The authors would like to thank the Brazilian National Agency of Petroleum Natural Gas and Biofuel (ANP), PRH-ANP, Coordination for the Improvement of Higher Education Personnel (CAPES), and Computer Modeling Group (CMG) for the support.

\section{NOMENCLATURE}

ATBS - Acrylamide tertiary butyl sulfonic acid AQD - Acceptable quadratic distance

$C_{p}$ - Constant to prevent error when the observed data is close to zero

EOR - Enhanced oil recovery
FR - Oil Recovery Factor

HPAM - Hydrolyzed polyacrylamide

IAPV - Inaccessible pore volume

LD - Linear distance

$\mathrm{N}$ - Number of observed data

NALD - Normalized absolute linear distance

$\mathrm{Np}$ - Cumulative Oil Production

NQD - Normalized quadratic distance

NQDS - Normalized quadratic distance with signal

Obs - Observed data

QD - Quadratic distance

Sim - Simulated data

Tol - Tolerance related to the observed data

Wcut - Cumulative Water Cut

Wi - Cumulative Water Injection

WOR - Cumulative Water-Oil Ratio

$W p$ - Cumulative Water Production

$\Delta p$ - Differential Pressure

$\eta_{\text {bulk }}$ - Polymer apparent bulk viscosity

$\eta_{\text {is }}$ - Polymer apparent in-situ viscosity

$\mu_{w}-$ Water viscosity

\section{REFERENCES}

Avansi, G. D.; Schiozer, D. J. UNISIM-I: Synthetic Model for Reservoir Development and Management Applications. International Journal of Modeling and Simulation for the Petroleum Industry, v. 9, n. 1, p. 21-30. 2015.

Aziz, K.; Settari, A. Petroleum Reservoir Simulation. London, UK: Applied Science Publishers, 1979.

Dawson, R.; Lantz, R. B. Inaccessible Pore Volume in Polymer Flooding. Society of Petroleum Engineers Journal, v. 12, n. 5, p. 448-452. 1972. https://doi.org/10.2118/3522-PA

de Ghetto, G.; Paone, F.; Villa, M. PressureVolume-Temperature Correlations for Heavy and Extra Heavy Oils. Proceedings SPE International Heavy Oil Symposium, Calgary, Canada, 19-21 June 1995. https://doi.org/10.2118/30316-MS

Delamaide, E. Polymer Flooding of Heavy Oil From Screening to Full-Field Extension. Proceedings SPE Heavy and Extra Heavy Oil Conference: Latin America, Mendellín, Colombia, 24-26 September 2014. https://doi.org/10.2118/171105-MS 
Dominguez, J. G.; Willhite, G. P. Retention and Flow Characteristics of Polymer Solutions in Porous Media. Society of Petroleum Engineers Journal, v. 17, n. 02, p. 111-121. 1977.

https://doi.org/10.2118/5835-PA

Ferreira, V. H. S.; Moreno, R. B. Z. L. SinglePhase Polymer Flow in Porous Media: Numerical Model for Experimental Planning and Analysis. International Journal of Modeling and Simulation for the Petroleum Industry, v. 10, n. 1, p. 11-20. 2017a.

Ferreira, V. H. S.; Moreno, R. B. Z. L. Impact of flow rate variation in dynamic properties of a terpolymer in sandstone. Journal of Petroleum Science and Engineering, v. 157, p. 737-746. 2017b. https://doi.org/10.1016/i.petrol.2017.07.071

Ferreira, V. H. S.; Moreno, R. B. Z. L. Workflow for Oil Recovery Design by Polymer Flooding. Proceedings ASME 2018 37th International Conference on Ocean, Offshore and Arctic Engineering, Madrid, Spain, 17-22 June 2018. https://doi.org/10.1115/OMAE2018-78359

Gaspar, A. T. F. S.; Avansi, G. D.; Maschio, C.; Santos, A. A. S.; Schiozer, D. J. UNISIM-I-M : Benchmark Case Proposal for Oil Reservoir Management. Proceedings SPE Trinidad and Tobago Section Energy Resources Conference, Port of Spain, Trinidad and Tobago, 13-15 June 2016. https://doi.org/10.2118/180848-MS

Johnson, E. F.; Bossler, D. P.; Naumann, V. O. Calculation of relative permeability from Displacement Experiments. Petroleum Transactions, AIME, v. 216, p. 370-372. 1959.

Lamas, L. F. O.; Schiozer, D. J.; Delshad, M. Impacts of polymer properties on field indicators of reservoir development projects. Journal of Petroleum Science and Engineering, v. 147, p. 346-355. 2016.

https://doi.org/10.1016/i.petrol.2016.05.020

Maschio, C.; Schiozer, D. J. Probabilistic history matching using discrete Latin Hypercube sampling and nonparametric density estimation. Journal of Petroleum Science and Engineering, v. 147, p. 98115. 2016.

https://doi.org/10.1016/i.petrol.2016.05.011
Seright, R. S.; Fan, T.; Wavrik, K.; Balaban, R. C. New Insights Into Polymer Rheology in Porous Media. SPE Journal, v. 16, n. 01, p. 35-42. 2011. https://doi.org/10.2118/129200-PA

Silveira, B. M. O. Projeto de Fluido e Testes de Deslocamento para Avaliação da Recuperação de Óleo Pesado por Injeção de Polímero. 140p. PhD Thesis. School of Mechanical Engineering, University of Campinas, Brazil, 2017. (in Portuguese)

Silveira, B. M. O.; Lopes, L. F.; Moreno, R. B. Z. L. Rheological Approach of HPAM Solutions under Harsh Conditions for EOR Applications. International Journal of Engineering \& Technology IJET-IJENS, v. 16, n. 03, p. 1-8. 2016.

Silveira, B. M. O.; Lopes, L. F.; Moreno, R. B. Z. L. Polymer Flooding in a High Salinity Heavy-Oil Reservoir. Brazilian Journal of Petroleum and Gas, v. 12, n. 1, p. 35-51. 2018.

https://doi.org/10.5419/bjpg2018-0004

Sorbie, K. S. Depleted Layer Effects in Polymer Flow through Porous Media. Journal of Colloid and Interface Science, v. 139, n. 2, p. 299-314. 1990. https://doi.org/10.1016/0021-9797(90)90103-U

Sorbie, K. S. Polymer-Improved Oil Recovery. Boca Raton, Florida, USA: Blakie, 1991. https://doi.org/10.1007/978-94-011-3044-8

Standnes, D. C.; Skjevrak, I. Literature Review of Implemented Polymer Field Projects. Journal of Petroleum Science and Engineering, v. 122, p. 761-775. 2014.

https://doi.org/10.1016/i.petrol.2014.08.024

Stavland, A.; Jonsbraten, H.; Lohne, A.; Giske, N. $\mathrm{H}$. Polymer Flooding - Flow Properties in Porous Media versus Rheological Parameters. Proceedings SPE EUROPEC/EAGE Annual Conference and Exhibition, Barcelona, Spain, 14-17 June 2010. https://doi.org/10.2118/131103-MS

Zampieri, M. F. Evaluation of Polymer Flooding through Small Scale Simulation Models for Enhanced Oil Recovery. 272p. PhD. Thesis. University of Campinas, 2017. 